\title{
Adaptation and validation of the ITC - Sense of Presence Inventory for the Portuguese language
}

\author{
José Vasconcelos-Raposo $^{\mathrm{a}, \mathrm{b}}$, Miguel Melo*,a ${ }^{*}$, Carla Teixeira ${ }^{\mathrm{b}}$, Luciana Cabral ${ }^{\mathrm{c}, \mathrm{d}}$, \\ Maximino Bessa ${ }^{\mathrm{a}, \mathrm{b}}$ \\ ${ }^{\text {a }}$ INESC TEC, Porto, Portugal \\ ${ }^{\mathrm{b}}$ Universidade de Trás-os-Montes e Alto Douro, Vila Real, Portugal \\ c Instituto Politécnico de Bragança, Bragança, Portugal \\ ${ }^{\mathrm{d}}$ CITCEM, Porto, Portugal
}

\section{A R T I C L E I N F O}

\section{Keywords:}

Virtual reality

Human-Computer interaction

Subjective evaluation

Presence

\begin{abstract}
A B S T R A C T
This investigation concerns the translation and validation of the ITC - Sense of Presence Inventory (ITC-SOPI) for the Portuguese-speaking population (in Europe), estimating the validity of the content and concepts and the maintenance of an equivalent semantics. It also sought to verify its psychometric properties, namely its factorial validity and internal consistency. The sample consisted of 459 individuals, 274 males and 185 females. The fidelity of the subscales varied between 0.67 and 0.89 . Confirmatory factor analysis revealed a theoretical model of 35 items, divided by four factors. After fixing some of the residual errors between items, the following adjustment indexes were calculated: $\chi^{2} / d f=2.301$; goodness fit index $=0.860$; comparative fitness index $=0.889$; root mean square error of approximation $=0.053$; Akaike's information criterion $=1420$. Based on the observed results and the robustness of the sample size used, the obtained theoretical model shows that the ITC-SOPI is recommended to measure presence in virtual reality research projects with samples of Portuguese language speakers.
\end{abstract}

\section{Introduction}

At present, virtual reality (VR) equipment allows its users to interact with a virtual simulation, which is often multisensory in character and aims at providing a total immersion of the senses (Saposnik et al., 2011; Schuemie et al., 2001).

The domains of applicability of VR extend over different areas of professional intervention, namely, in clinical situations, for the treatment of anxiety disorders (Cobb and Sharkey, 2007) and post-traumatic stress disorder (Rothbaum et al., 2001), in surgical training (Ahlberg et al., 2007; Gallagher et al., 2005; Seymour et al., 2002), in pain tolerance therapies (Czub and Piskorz, 2014), in sport contexts, for overcoming limitations associated with the performance of athletes (Bideau et al., 2010), and in military training and simulations (Taylor et al., 1997; Zyda, 2005).

To evaluate the experiences in VR, the notion of the sense of presence arises as fundamental in the evaluation and understanding of the way the user experiences these simulations, considering that an illusion is provided in which the user perceives himself to be located in a different place than his physical environment (Lessiter et al., 2001).
According to Lee (2004) and Schubert et al. (2001), the concept of presence is defined as a sequence in which the VR user becomes involved in the simulation environment or in the virtual stimuli that are presented to him. Lessiter et al. (2001) argue that the concept of presence encompasses the user's feelings of being spatially located in a given location within the simulated environment made available by the equipment and is characterized by a strong sensation associated with the dynamic perception-action between the equipment and the user. For the authors, the feeling of presence appeals to the naturalness and credibility of the content provided by the virtual environment.

One of the instruments used internationally to measure presence is referred to as the ITC - Sense of Presence Inventory (ITC-SOPI), developed by Lessiter et al. (2001), with the aim of providing the research community with a reliable and valid instrument that would allow results from different laboratories to be compared, thus contributing to the development and deepening of knowledge in this field.

The ITC-SOPI allows one to analyse user experience with VR technologies. It is divided into two parts, A and B, where the questions in part A relate to the user's feelings after the VR experience, and the questions in part $\mathrm{B}$ allow one to characterize the experience during the

\footnotetext{
* Corresponding author.

E-mail address: mcmelo@inesctec.pt (M. Melo).
} 
simulation.

Initially, the questionnaire consisted of 63 items, covering the content of several areas that the authors considered relevant for defining presence (i.e., sense of space, involvement, attention, distraction, autonomy, realism, naturalness, perception of time, awareness of actions, sense of social interaction, personal relevance, excitement and negative effects), with part A consisting of 7 items and part B consisting of 56.

After a series of factor analysis procedures, four factors were extracted: Sense of Physical Space (19 items), Engagement (13 items), Ecological Validity (5 items), and Negative Effects (6 items), Nineteen items were eliminated either because they did not meet the criteria of the factor analysis or they reduced the internal consistency of the factors.

Finally, the authors presented a revised and final version of the inventory with a total of 44 items divided into two parts. Part A, consisting of 6 items, and part B, consisting of 38 items, were organized into four factors: Sense of Physical Space, Engagement, Ecological Validity, and Negative Effects. Lessiter et al. (2001) characterized Sense of Physical Space as the feeling of being physically positioned in the simulated environment, encompassing the ability to physically control and manipulate details of the experience.

Engagement refers to the psychological involvement, interest, and user satisfaction with the content of the experience. Ecological Validity refers to the credibility or realism of the content and the naturalness and consistency of the simulated environment, that is, how realistic or real the user perceived the virtual environment to be.

Finally, Negative Effects encompass items that describe the adverse psychological reactions of exposure to the virtual experience. According to the authors, the four factors were probably established due to the interaction between the elements that give form and content to the experience owing to the personal characteristics of the user.

The lack of psychometric instruments translated and validated in VR research using Portuguese language-speaking samples led to the main objective of this research, namely, the translation and validation of the ITC-SOPI for the Portuguese language (Europe). This adaptation takes into account the need to preserve semantic, conceptual and content equivalence and validity for each item.

The main objective is to investigate the psychometric properties of the instrument using descriptive statistics and confirmatory factor analysis techniques, with the aim of making available the ITC-SOPI for research projects on presence in VR contexts.

\section{Material and methods}

The sample comprised 459 individuals, with 274 male individuals and 185 female individuals aged between 17 and 56 years $(M=24.54$; $S D=6.7)$. Five participants with vision problems and showing nausea were eliminated. Participants were recruited from two higher education institutions using a non-probabilistic (convenience) sampling technique.

Participants shared similar social and cultural milieus, with corresponding life experiences. That is, the vast majority did not need vision or hearing aids $(61.2 \%$ and $99.3 \%$, respectively), and $81.4 \%$ of participants had prior information about VR equipment.

No significant differences regarding this knowledge were found. Most participants had never previously used VR glasses (82.8\%) nor had previously participated in an experiment similar to that in this study (95.6\%). The participants were familiar with the use of computers and could be classified on three different levels: basic (11.2\%), intermediate $(26.6 \%)$ and good $(62.3 \%)$.

Finally, most participants mentioned that they used computers recreationally from 1 to 2 times (38.6\%), 3 to 4 times (15.7\%) or 5 to 6 times $(10.7 \%)$ a week or everyday (13.7\%). All participants came from similar sociocultural backgrounds, with similar life experiences. Thirteen participants ( $2.8 \%$ of the sample) dropped out of the study but did not present any threat to internal validity.

\subsection{Instruments}

Questionnaire: The ITC-SOPI is characterized as a 44-item selfreport questionnaire developed by Lessiter et al. (2001). It is divided into two parts, A (6 items) and B (38 items), with questions that address the individual's feelings after the VR experience and questions that describe the experience during virtual simulation, respectively. This instrument is divided into 4 subscales: Sense of Physical Space (19 items), Engagement (13 items), Ecological Validity (5 items), and Negative Effects ( 6 items). The question responses are presented on a fivepoint Likert scale ( $1=$ strongly disagree, $5=$ strongly agree).

Translation and cultural adaptation: The methodology adopted for the translation process was the back-translation method (Brislin, 1970; Hambleton and Zenisky, 2011). The ITC-SOPI in its original version was translated and re-translated by a total of four doctoratelevel bilinguals: two with $\mathrm{PhDs}$, with specialization in psychometrics, and two PhDs in the field of Informatics, specializing in VR. One researcher translated the ITC-SOPI from the English language into the Portuguese language. Then, another researcher completed the retroversion without consulting the original version. The version used in the present study was attained following this procedure (Freire and Almeida, 2008; Hambleton and Zenisky, 2011). The same researchers then looked at the validity of the content. Thus, they individually mentioned whether they agreed or disagreed with the inclusion of the items in the originally proposed factors.

Subsequently, they calculated the value of agreement of the items in the respective factors based on a 10-point scale $(1=$ not relevant, $10=$ extremely relevant). The content validity index (Waltz et al., 1991)was determined, with results above $80 \%$ for all items, which supported their inclusion in the respective factors.

The institutional authorities (e.g., ethics committees) approved the project and gave permission to collect the data. All the participants were asked to sign a consent form to formalize their agreement to participate in the study. A debriefing session was completed with each participant in which the research team disclosed the objectives of the research and how they would participate in the study. They were also informed that at any moment, they could quit the study. Sociodemographic data were collected and processed in a manner that ensured participants' anonymity and the confidentiality of the data.

Virtual environment: Participants experienced a virtual simulation in which they were presented different stimuli that allowed them to develop a state of presence. This experience enabled them to complete the ITC-SOPI after exposure to the stimulus. One used the VR application "Don't let go!" (Skydome Studios, 2014), which consists of a gamestyle application of a first-person experience in which the user is behind a desk and has to press both control keys (Ctrl) keys on the keyboard. The goal of the game is to press and hold both control keys throughout the whole experience. During the experiment, a series of events arise, the objective of which is to produce fear and/or stress and to cause the release of the keys. Examples of such events that occur in the virtual environment are the appearance of a swarm of bees to disturb the participant, the sudden drop of knives near the participant's virtual hands or spiders walking along the virtual arms of the participant (illustrated in Fig. 1).

For the virtual simulation, we used an Asus N550JK-CN104H laptop equipped with an Intel Core i7 4700HQ CPU, an NVIDIA GeForce GTX850M graphics card with a 4GB DDR3 memory card, and 16GB of RAM, together with an SSD disk.

In the test, participants had in front of them a Microsoft Wired 600 keyboard that was not connected to the laptop. The headphones used were the Bose QuietComfort 15 model, which allowed fir very effective insulation of acoustic noise. The HMD used was the Oculus Rift DK2. Still, it was possible to apply an FHD resolution $(1920 \times 1080)$ with an average image frequency of 60 FPS. 


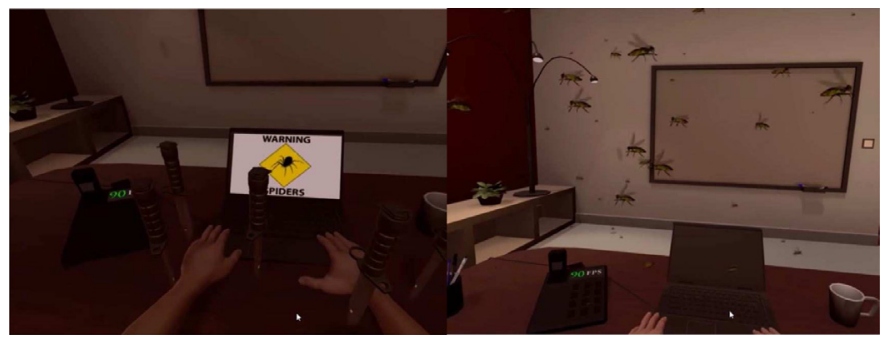

Fig. 1. Perspectives of the participants in the VR application.

Experimental procedure: Each experimental procedure took approximately $15 \mathrm{~min}$. Each virtual experience lasted roughly $3 \mathrm{~min}$ and $45 \mathrm{~s}$. In the room provided for the experiments, a cabin was installed that isolated the participants from the outside environment during the tests. Inside of it was placed a desk, a keyboard, a pair of headphones and the Oculus Rift. Participants were instructed to use the equipment correctly. At the end of the virtual experiment, participants were told to go to the room where they completed the ITC-SOPI.

Statistical procedures: Descriptive statistics were performed for all factors and their respective items (means and standard deviations), and the coefficients of univariate normality (skewness and kurtosis) were also calculated in order to ascertain the symmetry of the distribution. Cronbach's $\alpha$ was calculated to confirm the internal consistency of the data. The presence of outliers was studied by calculating the Mahalanobis squared distance $\left(D^{2}\right)$, using SPSS (SPSS v.21, IBM Company, Chicago, IL).

Confirmatory factor analysis (Amos 24.0, SPSS, At IBM Company, Chicago, IL) was performed to validate the ITC theoretical model originally proposed by Lessiter et al., 2001), and the adequacy of the structure was examined using the obtained adjustment indexes. The $\chi$ test (chi-square) identifies a non-significant adjustment value $(p>0.05)$. However, the results of this test are questionable, as it is conditioned by the size of the sample. To overcome such a limitation, we used a corrective procedure, calculating the ratio of the chi-square to its corresponding degrees of freedom $\left(\chi^{2} / d f\right)$ (Jöreskog and Sörbom, 1996). Marôco (2014) considers it possible to accept the theoretical model when $\chi^{2} / d f \leq 2.0$.

Other adjustment quality indexes have also been used, such as the CFI (comparative fit index) and GFI (goodness of fit index), which produce values within the range 0 to 1 . Results above 0.90 represent an appropriate model (Bentler and Bonett, 1980; Marôco, 2014), with values $\geq 0.95$ indicating a very good fit of the model ( $\mathrm{Hu}$ and Bentler, 1999; Marôco, 2014). Finally, the RMSEA (root mean square error of approximation) index was used, with low values demonstrating an acceptable model fit.

Specifically, the RMSEA should be equal to or less than 0.10 , with 0.05 considered representative of a very good fit (Marôco, 2014), although Browne and Cudeck (1992) argued that the result should be equal to or less than 0.08, whereas Hu and Bentler (1999) argued that it should be equal to or less than 0.06 . The composite reliability and the mean-variance extracted from each factor were calculated using the formula proposed by Marôco (2014).

\section{Results}

To simplify the analysis, only the descriptive statistics (means and standard deviations) and the univariate normalities (skewness and kurtosis) regarding the factors of the instrument were described, as seen in Table 1. The coefficients of skewness $(S K)$ and kurtosis $(K U)$ were calculated for each item, with values varying between -2.841 and 1.793 and -1.007 and 9.602, in this order, certifying the normal distribution of the sample $(|S K|<3$ and $|K U|<10)$ Marôco, 2014).

In the analysis of the internal consistency of the data, it was possible to verify that the Physical Space factor had good internal consistency,
Table 1

Descriptive and univariate analyses of the normality and Cronbach's $\alpha$ of the factors.

\begin{tabular}{lllll}
\hline & $M \pm S D$ & Skewness & Kurtosis & $\alpha$ \\
\hline Sense of physical space & $65.23 \pm 10.18$ & -0.379 & 0.250 & 0.89 \\
Engagement & $21.64 \pm 3.81$ & -0.302 & 0.175 & 0.67 \\
Ecological Validity & $17.38 \pm 3.62$ & -0.331 & 0.036 & 0.79 \\
Negative effects & $11.46 \pm 4.25$ & 0.628 & -0.262 & 0.78 \\
\hline
\end{tabular}

with a value higher than 0.80 . All other factors exhibited acceptable internal consistency, with scores greater than 0.50 and less than 80 . The total internal consistency of the ITC-SOPI was good, with a value higher than $0.80(\alpha=0.909)$.

The sample was homogeneous, as all the subjects shared sociocultural characteristics and had similar life paths, and it was verified that $81.4 \%$ of them reported being familiar with VR equipment. However, this contact was primarily related to knowledge of the technology in general and not to the use of it.

\subsection{Confirmatory factor analysis}

The factorial validity of the ITC was estimated through confirmatory factor analysis using AMOS-24 software, as described in Marôco (2014). No outliers were identified, nor were any variables whose Sk and Ku coefficients suggested an abnormal distribution $(|S K|<3$ and $|K U|<10$ see Marôco, 2014).

The overall fit of the factorial model was assessed using the indexes and standard values reported by Marôco (2014): $\chi^{2} / d f$, CFI, GFI, RMSEA, $P$ [RMSEA $\leq 0.05$ ] and MECVI. The quality of the local fit was evaluated based on the factorial weights and the individual reliability of the items. Adjustment of the model was performed based on modification indexes above 11 , with $p<0.01$, calculated using AMOS24 and obeying the theoretical considerations described in the literature (Kline, 2015; Marôco, 2014).

The confirmatory factor analysis allowed for the evaluation of the theoretical model of 44 items, with four subscales (Sense of Physical Space, Engagement, Ecological Validity and Negative Effects), developed by Lessiter et al. (2001). In this sense, the necessary statistical procedures were performed to calculate the adjustment indexes of the model.

In the analysis of the selected indexes to determine the suitability of the model, the following values were considered as reference values to indicate acceptable fit: $\chi^{2} / d f \mathrm{df}$ in the range of 1 to 2 ; GFI > 0.90; CFI $>0.90$; and RMSEA, $<0.10$ (Marôco, 2014). Based on the output of the confirmatory factor analysis from AMOS-24, we proceeded to a set of modifications that made it possible to improve the theoretical model.

In the first test of the original ITC-SOPI version with 44 items, the results indicated an inadequate fit $\left(\chi^{2} / d f=4.237\right.$, GFI $=0.671$, CFI $=0.645$, RMSEA $=0.084, P$ [RMSEA $\leq 0.05]$, MECVI $=8345$ ). No outliers were identified, and therefore, no observations of the model were eliminated. However, a set of corrections were suggested, and consequently, measurement errors were correlated for each of the subscales as follows:

- Sense of physical space: e1-e15, e1-e18, e4-e17, e7-e9, e7-e15, e7-e12, e8-e9, e8-e13, e8- e15, e8- E8-e19, e10-e11, e10-e15, e11-e18, e13-e15, e13-e18, e15-e18, e16-e17, e16-e19;

- Engagement: e20-e22, e20-e28, e21-e26, e22-e28, e23-e33, e23-e28, e24-e25, e24-e33, e25-e27, e27-e30, e27-e31, e28 -e33, e30-e32;

- Negative effects: e39-e41, e40-e41.

After these corrections were made, a satisfactory improvement of the adjustment indexes $\left(\chi^{2} / d f=2.531\right.$, GFI $=0.817$, CFI $=0.839$, 
RMSEA $=0.058, P$ [RMSEA $\leq 0.05]$, MECVI $=5140$ ) was observed. Then, eight items ( 8 items belonging to the Engagement factor and 1 item belonging to the Sense of Physical Space factor) were removed. The criteria used in the removal were based on the exclusion of items that had a factorial load lower than 0.4 and the existence of modification indexes that indicated a saturation of items in factors other than those proposed in the original version of the instrument. With these corrections, the following indicators were calculated, indicating an acceptable overall fit of the model: $\chi^{2} / d f=2.180$, GFI $=0.871$, $\mathrm{CFI}=0.898, \mathrm{RSMEA}=0.051, \mathrm{AIC}=1280.086$, and MECVI $=2.829$.

The composite reliability of each factor was calculated and the following values were determined: Physical Space Sense (SPS) $=0.891$; Engagement $(\mathrm{E})=0.672$, Ecological Validity $(\mathrm{EV})=0.802$, and Negative Effects $(\mathrm{NE})=0.765$. These results reveal that all factors present good composite reliability.

The discriminant validity of the factors was verified by comparing the values of the average variance extracted (AVE) with the square of the correlation between the factors $\left(r^{2}\right)$ (Marôco, 2014). Discriminant validity is ensured when the AVE values of each factor are higher than the squares of their correlations. The values of the mean extracted variance varied between 0.276 and 0.454 . By contrasts, $r^{2}$ values ranged from 0.001 to 0.342 . Thus, by comparing the AVE values with the squares of the correlations between the factors, it was verified that there is a three-factor discriminant validity, except for the Engagement factor, for which the AVE value (AVEC $=0.276$ ) was lower than the square of the correlation with the Ecological Validity factor $\left(r_{\mathrm{C}-\mathrm{VE}}^{2}=0.342\right)$.

The final theoretical model consisted of 37 items divided into 4 factors (Sense of Physical Space with 18 items, Engagement with 5 items, Ecological Validity with 5 items and Negative Effects with 6 items), as seen in Fig. 2.

\section{Discussion}

Psychometric instruments in their original versions are developed in a given cultural and linguistic context. As such, they need to be translated and validated in order to be applied to other contexts. There is currently a lack of instruments validated for the Portuguese language that allow for the evaluation of perception and satisfaction during virtual simulation experiments. Thus, it is urgent that this situation be corrected with a study that aims at overcoming this limitation by validating instruments for the Portuguese language that make it possible to measure the human experience in VR tests.

Thus, the present study had as a main objective to translate and validate the original version of the ITC-SOPI (Lessiter et al., 2001) for the Portuguese language. For this purpose, we used a sample of 459 university students from northern Portugal. To adapt the instrument, specific instrument validation techniques (e.g., semantic and content analysis of the items) were carried out, which allowed for an improvement in the validity of the constructs and their internal consistency. The application of the AFC was carried out to preserve the original structure of the theoretical model. That is, the same numbers of factors and items were preserved in order to fulfill the assumptions of the validation theory (Cheung et al., 2011).

The execution of these procedures facilitated correct validation of the theoretical model, maintaining the concepts and content proposed in the original version. Consequently, it is possible to apply this instrument to another sociocultural context given that the interpretation criteria are ensured.

A comparison between the proposed model (the original version of ITC-SOPI) and that obtained with our sample (the final version obtained by us) shows that it is possible to retain the four initial factors. However, the Portuguese version (Europe) consists of 35 items, not 44, as initially proposed. In the version validated by us, eight items ( 3 items from part A and 5 items from part B) were removed. Thus, part A now consists of only 2 items, whereas part B has 33 items. The modifications made through the confirmatory factor analysis allowed us to reach satisfactory global fit values regarding the indexes and validity.

Discriminant validity was observed for most of the factors except for the Engagement factor, which presented a VME value lower than the square of the correlations between this factor and the Ecological Validity factor. Regarding the results of the extracted mean variance, values lower than the desirable ones were obtained for all factors. According to Valentini and Bruno (2017), the values of VME must be equal to or greater than 0.50 in order to be taken as indicators of an adequate fit of the model. However, others suggest that a comparison of the dimension of VMS values with those of the square of the correlation between the factors in question be used, which is what we did in this study.

Examination of the internal consistency of the factors indicated that a higher alpha value (alpha $=0.89$ ) was obtained in our sample in comparison to the original model of the Physical Space factor (alpha $=0.88$ ). However, in the Engagement factor, the calculated alpha value was lower (alpha $=0.67)$ compared to the original version of this factor (alpha $=0.76$ ). For the remaining two factors, the alpha values were preserved.

Considering the results obtained (fit indexes, validity and internal consistency) through the confirmatory factor analysis and the descriptive analysis, as well as the adequate sample size used, the final version of the ITC-SOPI for the Portuguese language (Europe) that we achieved is an acceptably robust instrument that can be used in VR research projects with samples of Portuguese language speakers. Nevertheless, the present study was based only on a single VR experience; therefore, we plan to extend this validation to different VR applications to improve the robustness of this instrument.

\section{Conclusions}

Overall, the results of the present study revealed that with a reduction from 44 to 35 items, while keeping the four factors initially proposed, the ITC-SOPI presents a reliable measure to assess the Sense of Physical Space, Engagement, Ecological Validity and Negative Effects in studies on presence in VR. ${ }^{1}$

However, other studies using this validated version of the ITC should be conducted to confirm the validity and reliability of the factorial structure that we obtained. It would be desirable for subsequent studies to recruit a larger number of participants and compare individuals with experience in the use of VR equipment with others without such experience.

\section{Acknowledgments}

This work is financed by the ERDF European Regional Development Fund through the Operational Programme for Competitiveness and Internationalisation - COMPETE 2020 Programme and by National Funds through the Portuguese funding agency, FCT - Fundação para a Ciência e a Tecnologia within project POCI-01-0145-FEDER-028618 entitled PERFECT - Perceptual Equivalence in virtual Reality For authEntiC Training. All the works were conducted at INESC TEC's MASSIVE Virtual Reality Laboratory.

\section{Conflict of interest}

i2 media research holds the copyrights of the original ITC-SOPI questionnaire and a distribution agreement was reached. However, the i2 media research group was in no way involved in data analysis or

\footnotetext{
${ }^{1}$ To use the Portuguese version of ITC-SOPI it is required to ask for permission to the authors that will make available the questionnaire once they grant permission.
} 


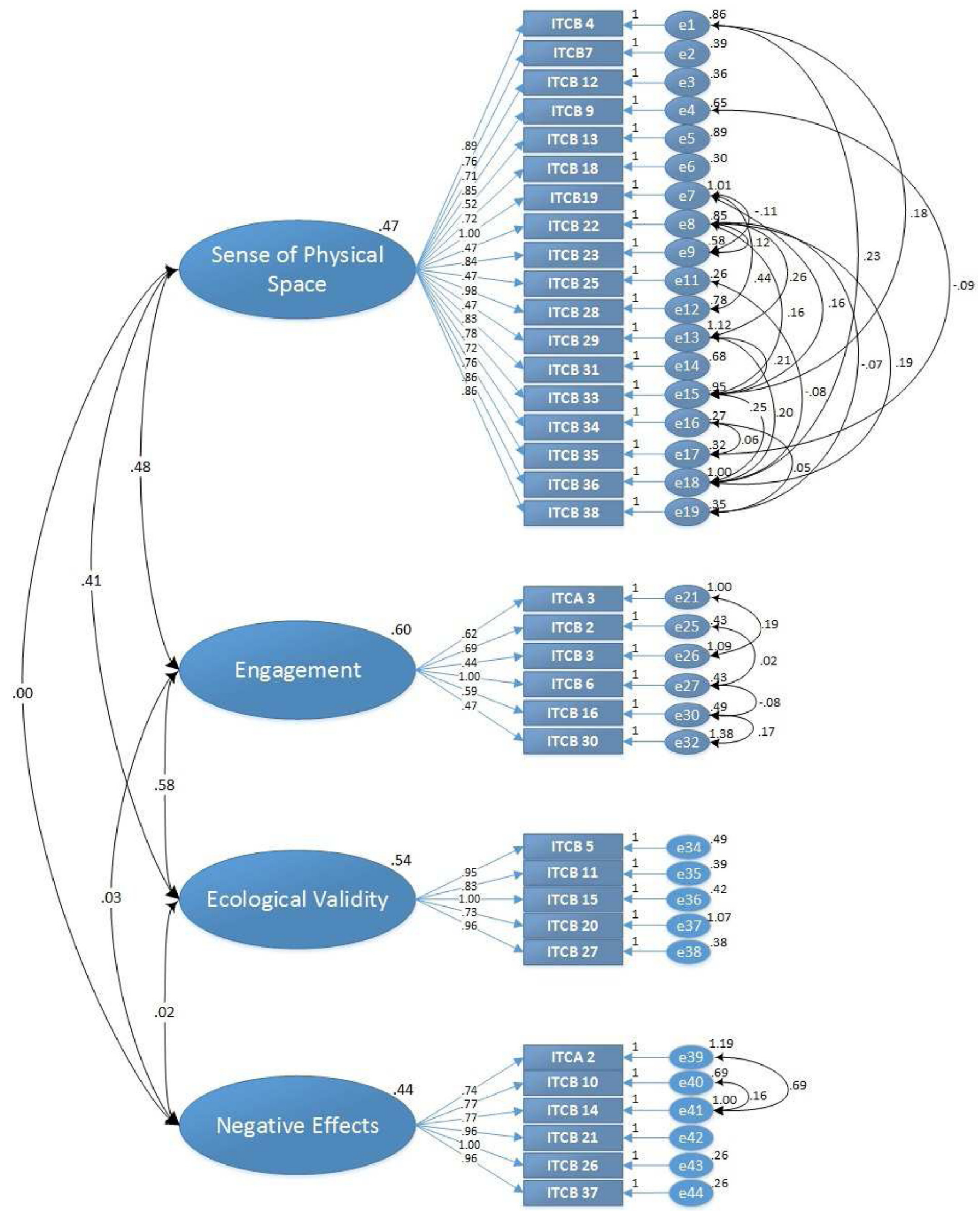

Fig. 2. Theoretical model of the ITC-SOPI adapted in Portuguese. 
conclusions of this paper. There are no other known conflicts of interest associated with this publication.

\section{References}

Ahlberg, G., Enochsson, L., Gallagher, A.G., Hedman, L., Hogman, C., McClusky, D.A., Ramel, S., Smith, C.D., Arvidsson, D., 2007. Proficiency-based virtual reality training significantly reduces the error rate for residents during their first 10 laparoscopic cholecystectomies. Am. J. Surg. 193, 797-804.

Bentler, P.M., Bonett, D.G., 1980. Significance tests and goodness of fit in the analysis of covariance structures. Psychol. Bull. 88, 588.

Bideau, B., Kulpa, R., Vignais, N., Brault, S., Multon, F., Craig, C., 2010. Using virtual reality to analyze sports performance. IEEE Comput. Graph. Appl. 30, 14-21.

Brislin, R.W., 1970. Back-translation for cross-cultural research. J. Cross Cult. Psychol. 1, $185-216$.

Browne, M.W., Cudeck, R., 1992. Alternative ways of assessing model fit. Sociolog. Methods Res. 21, 230-258.

Cheung, F.M., van de Vijver, F.J., Leong, F.T., 2011. Toward a new approach to the study of personality in culture. Am. Psychol. 66, 593.

Cobb, S., Sharkey, P.M., 2007. A decade of research and development in disability, virtual reality and associated technologies: review of icdvrat 1996-2006. IJVR 6, 51-68.

Czub, M., Piskorz, J., 2014. How body movement influences virtual reality analgesia? Interactive Technologies and Games (iTAG), 2014 International Conference on. IEEE, pp. 13-19.

Freire, T., Almeida, L.S., 2008. Metodologia da Investigação em Psicologia e Educação. Gallagher, A.G., Ritter, E.M., Champion, H., Higgins, G., Fried, M.P., Moses, G., Smith, C.D., Satava, R.M., 2005. Virtual reality simulation for the operating room: proficiency-based training as a paradigm shift in surgical skills training. Ann. Surg. 241, 364-372.

Hambleton, R., Zenisky, A.L., 2011. Translating and adapting tests for cross-cultural assessments. Cross-Cultural Research Methods in Psychology. pp. 46-70.

Hu, L.-t., Bentler, P.M., 1999. Cutoff criteria for fit indexes in covariance structure analysis: conventional criteria versus new alternatives. Struct. Equ. Model. 6, 1-55. Jöreskog, K.G., Sörbom, D., 1996. LISREL 8: User's Reference Guide. Scientific Software International.

Kline, R.B., 2015. Principles and Practice of Structural Equation Modeling. Guilford publications.

Lee, K.M., 2004. Presence, explicated. Commun. Theory 14, 27-50.

Lessiter, J., Freeman, J., Keogh, E., Davidoff, J., 2001. A cross-media presence questionnaire: the itc-sense of presence inventory. Presence: Teleoper. Virtual Environ. 10, 282-297.

Marôco, J., 2014. Análise de Equações Estruturais: Fundamentos teóricos, Software \& Aplicações, Second ed. Pêro Pinheiro.

Rothbaum, B.O., Hodges, L.F., Ready, D., Graap, K., Alarcon, R.D., 2001. Virtual reality exposure therapy for vietnam veterans with posttraumatic stress disorder. J. Clin. Psychiatry.

Saposnik, G., Levin, M., Outcome Research Canada (SORCan) Working Group, et al., 2011. Virtual reality in stroke rehabilitation. Stroke 42, 1380-1386.

Schubert, T., Friedmann, F., Regenbrecht, H., 2001. The experience of presence: factor analytic insights. Presence: Teleoper. Virtual Environ. 10, 266-281.

Schuemie, M.J., Van Der Straaten, P., Krijn, M., Van Der Mast, C.A., 2001. Research on presence in virtual reality: a survey. CyberPsychol. Behav, 4, 183-201.

Seymour, N.E., Gallagher, A.G., Roman, S.A., Obrien, M.K., Bansal, V.K., Andersen, D.K., Satava, R.M., 2002. Virtual reality training improves operating room performance: results of a randomized, double-blinded study. Ann. Surg. 236, 458-464.

Skydome Studios, 2014. Dont let go! (version 1.2). http://skydomestudios.nl/dont-let-go/ Accessed on 23-04-2018.

Taylor, H.L., Lintern, G., Hulin, C.L., Talleur, D., Emanuel, T., 1997. Transfer of Training Effectiveness of Personal Computer-Based Aviation Training Devices. Technical Report. DTIC Document.

Valentini, F., Bruno, D.F., 2017. Variância média extraída e confiabilidade composta: indicadores de precisão. Psicologia: Teoria e Pesquisa 32.

Waltz, C.F., Strickland, O., Lenz, E.R., 1991. Measurement in Nursing Research. FA Davis Company.

Zyda, M., 2005. From visual simulation to virtual reality to games. Computer 38, 25-32. 